TH6B-3

\title{
Ultra-Sensitive Microwave Detection of Protein Conformational Changes
}

\author{
Kimberly M. Taylor and Daniel W. van der Weide \\ Biophysics Degree Program and Department of Electrical \& Computer Engineering, University of \\ Wisconsin-Madison, Madison, WI 53706, USA
}

\begin{abstract}
A coaxial driven planar slot antenna resonant in the microwave regime was used to detect changes in protein conformation induced by temperature modulation. Simultaneous dielectric and UV/VIS spectroscopy measurements were obtained by attaching the slot antenna to a fusedquartz UV/VIS sample holder. Similar thermodynamic parameters (midpoint temperature and enthalpy) were obtained from both techniques over a wide concentration range and under a variety of $\mathrm{pH}$ conditions. Here we demonstrate that a near-field antenna can be used to detect changes in conformation at very low protein concentrations, and that the protein is not destabilized by the presence of microwave power.
\end{abstract}

Index Terms - Proteins, slot antennas, biochemistry, biophysics, biomedical applications of electromagnetic radiation

\section{INTRODUCTION}

Several methods are currently employed for the study of conformational changes of biological macromolecules in solution, including ultraviolet/visible (UV/VIS), circular dichroism, and fluorescence spectroscopies and several varieties of microcalorimetry. With the exception of fluorescence spectroscopy, all of these techniques require large concentrations of macromolecule; fluorescence spectroscopy uses only small amounts of reagent, but requires the presence (or the addition) of an appropriate fluorescent agent. Dielectric spectroscopy has been seldom applied to the study of biological molecules, but our variation on this technique shows promise for sensitive detection over a wide range of conditions.

The current work establishes a new application for dielectric spectroscopy in which proteins or other biomolecules are studied in an aqueous environment and in which UV/VIS spectroscopy is simultaneously performed. This method not only allows measurement of proteins and other biomolecules in their "natural" aqueous state, but also permits correlation and comparison with results obtained using an established method.

We employ a coaxial driven planar slot antenna resonant at approximately $3 \mathrm{GHz}$ when loaded with a fusedquartz cuvette and aqueous solution. Although proteins experience rotational orientation (called the $\beta$-dispersion
[1]) in the $\mathrm{kHz}$ to $\mathrm{MHz}$ range, this protein dispersion is overwhelmed by the stronger relaxation of water. At room temperature, bulk water possesses a dispersion over a very broad range centered at $19.2 \mathrm{GHz}$ ( $\gamma$-dispersion [1]). A protein or other biological molecule (e.g. nucleic acid or lipid) in aqueous solution is surrounded by a one or more layers of "bound" water molecules; these bound waters are rotationally and translationally hindered in comparison with bulk water and undergo dispersion at lower frequencies ( $\delta$-dispersion [1]). We use bound water as a reporter for the conformation or activity of a biological molecule. By focusing on the low $\mathrm{GHz}$ region, changes in $\delta$-dispersion, and thus changes in conformation, are detected as changes in the resonance of the slot antenna.

The slot antenna was attached to a fused-quartz cuvette suitable for UV/VIS spectroscopy. This enables simultaneous detection of dielectric and UV/VIS data, allowing correlation of changes in the resonance of the slot antenna with an established method.

Equilibrium thermal unfolding and refolding of the bovine pancreatic ribonuclease (RNase $A$ ) was used as a test system. RNase A is a small globular protein that undergoes reversible unfolding over a variety of buffer, salt and pH conditions [2]. Unfolding of a protein from the tightly folded native conformation to the more loosely packed, less ordered unfolded state is one of the largest conformational changes a protein can exhibit, and causes a large rearrangement of the water distribution around the protein. Thus, equilibrium thermal unfolding/refolding is a good target system for the investigation of detection of protein conformational change.

\section{EXPERIMENTAL SETUP}

The slot antenna was cut from RO-4003C, a highfrequency laminate (Rogers Corporation). The slot length was chosen to yield a principal resonance at approximately $3 \mathrm{GHz}$. The antenna was driven by a semi-rigid coaxial cable soldered across the center of the slot. Return loss was measured using a Hewlett-Packard 8720D vector network analyzer. 
RNase A solutions were prepared in one of the two buffers: 1) $50 \mathrm{mM}$ glycine/ $\mathrm{HCl}, 100 \mathrm{mM} \mathrm{NaCl}$ for $\mathrm{pH} 2-$ 3.5 ; or 2) $50 \mathrm{mM}$ sodium acetic/acetic acid, $100 \mathrm{mM} \mathrm{NaCl}$ for $\mathrm{pH}$ 4-5. All solutions were dialyzed exhaustively before use. Protein concentration was determined by absorbance at $278 \mathrm{~nm}$ for concentrations above $1 \mu \mathrm{M}$ [2]; lower concentrations were measured using a modified Lowry assay [3] (Pierce Biotechnology, Inc.). Thermal unfolding was monitored spectrophotometrically at 288 nm [2] using a Hewlett-Packard 8452A diode-array spec-

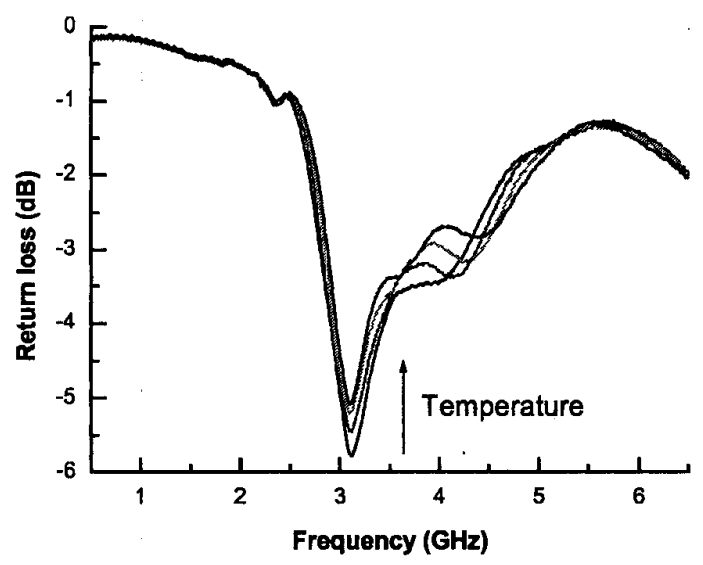

Figure 1: Representative return loss spectra for $78 \mu \mathrm{M}$ RNase A at $\mathrm{pH}$ 3.5. From bottom to top, the temperatures are 19.78, $39.55,59.30$ and $78.96^{\circ} \mathrm{C}$.

trophotometer.

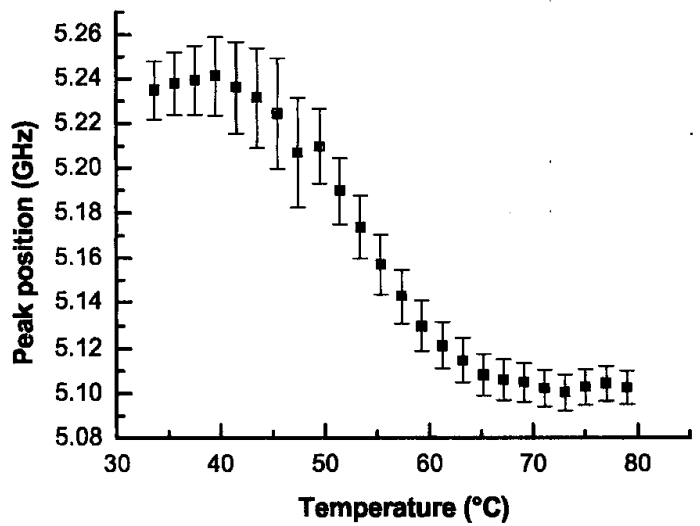

Figure 2: Variation in peak position vs. temperature for a selected peak. Protein concentration and $\mathrm{pH}$ are the same as in figure 1. Error bars indicate the uncertainty in peak position from the fit of the return loss spectrum to Lorentzians.

\section{ANALYSIS AND RESULTS}

\section{A. Return loss spectra and analysis}

Representative return loss spectra recorded at selected temperatures for a solution of $78 \mu \mathrm{M}$ RNase $\mathrm{A}$ in $50 \mathrm{mM}$ glycine/hydrochloric acid, $100 \mathrm{mM} \mathrm{NaCl}, \mathrm{pH} 3.5$ are shown in Figure 1. The general shape of the return loss spectra was invariant: a large resonance at approximately $3 \mathrm{GHz}$, with multiple side resonances. The spectra displayed temperature-dependent changes in both amplitude and frequency. Unfolding was $100 \%$ reversible (data not shown).

Return loss spectra were analyzed by fitting the spectrum at each temperature to a fixed number of Lorentzian peaks. The temperature-dependent behavior of a single peak could then be observed by plotting its position versus temperature. Some peaks exhibited sigmoidal temperature-dependent behavior characteristic of cooperative protein unfolding (see Figure 2). These sigmoidal peaks were subjected to a baseline-free analysis [4] that yielded midpoint temperature $\left(T_{m}\right.$, defined as the temperature at which $50 \%$ of the protein molecules are unfolded) and van't Hoff enthalpy $\left(\Delta \mathrm{H}_{\mathrm{m}}\right.$, defined as the heat released during the unfolding event, or the heat absorbed during refolding). These values of $T_{m}$ and $\Delta H_{m}$ are very similar to those obtained from concurrent spectrophotometric assays of unfolding/refolding (see Figure 3).

The sigmoidal peaks observed in protein solutions are absent when buffer is heated and cooled under the same conditions (data not shown). We conclude that the sigmoidal peak behavior is a reflection of protein conformational change, and not an artifact of the experimental conditions.

\section{B. Concentration dependence}

Unfolding/refolding assays at concentrations from $19 \mathrm{pM}$ to $680 \mu \mathrm{M}$ were performed. For concentrations above $8.8 \mu \mathrm{M}$, simultaneous UV/VIS absorbance was also recorded; the absorbance was too small for accurate measurement below $8.8 \mu \mathrm{M}$. These experiments served two purposes: 1) to test for microwave-related destabilization at low concentrations; 2) to establish the limits of sensitivity of the slot antenna system.

Since RNase A is a monomeric protein known to undergo two-state unfolding, no concentration-related variation of $T_{m}$ or $\Delta H_{m}$, such as would occur with multimeric proteins, was expected. Any concentration-related variation in these thermodynamic parameters was therefore due to the effects of microwave power.

The average midpoint temperature over this concentration range was $52.25 \pm 3.44^{\circ} \mathrm{C}$. No concentration-depen- 


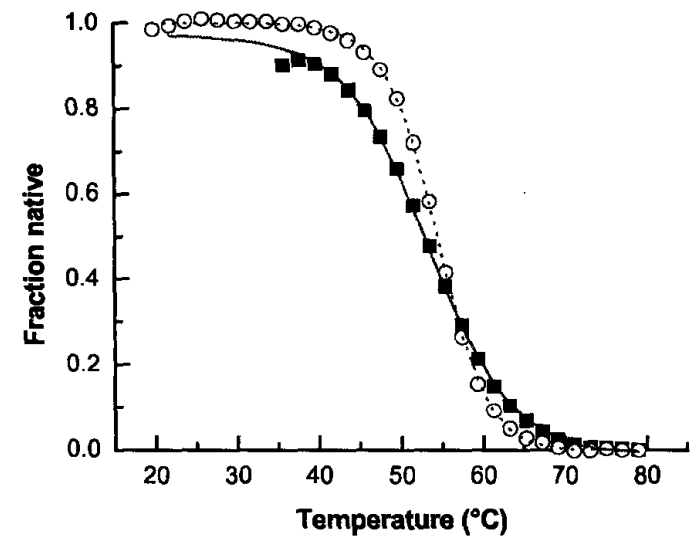

Figure 3: Analysis of peak positions from Figure 2 (solid symbols) and UV/VIS absorbance data (open symbols). Thermodynamic parameters from the peak position data were $T_{m}$ equals $51.38 \pm 1.37^{\circ} \mathrm{C}$ and $\Delta \mathrm{H}_{\mathrm{m}}$ equal to $47.67 \pm 5.83 \mathrm{kcal} / \mathrm{mol}$. For the UV/VIS data, $\mathrm{T}_{\mathrm{m}}$ was $53.44 \pm 0.60^{\circ} \mathrm{C}$ and $\Delta \mathrm{H}_{\mathrm{m}}$ was $67.35 \pm$ $3.00 \mathrm{kcal} / \mathrm{mol}$.

dent increase or decrease in $T_{m}$ was detected. The large error is probably due to increased experimental error at low concentrations. The average van't Hoff enthalpy was $50.83 \pm 4.81 \mathrm{kcal} / \mathrm{mol}$. These figures compare well with midpoint temperature $\left(54.01 \pm 0.57{ }^{\circ} \mathrm{C}\right)$ and enthalpy $(73.39 \pm 8.54 \mathrm{kcal} / \mathrm{mol})$ determined under the same conditions from UV/VIS spectroscopy. Thus we observed reliable data at concentrations several orders of magnitude lower than could be observed using standard methods such as UV/VIS or circular dichroism spectroscopy.

\section{C. $p H$ dependence}

The midpoint temperature and enthalpy of RNase A are known to decrease with $\mathrm{pH}$ for $\mathrm{pH}$ values in the range 2.5 to 5 [5]. This pH series fulfills two purposes: 1) to provide more evidence that the apparent dependence of peak position on protein conformation is not an effect limited to the specific conditions used in the previous experiments; 2) to allow estimation of the accuracy of the enthalpy measurements through determination of the heat capacity, $\Delta \mathrm{C}_{\mathrm{p}}$, defined as the slope of $\Delta \mathrm{H}_{\mathrm{m}} \mathrm{vs}$. $\mathrm{T}_{\mathrm{m}}$.

Values of $T_{m}$ and $\Delta H_{m}$ determined for UV/VIS and concurrent return loss measurements are shown in Table I. The expected $\mathrm{pH}$-dependent variation in thermodynamic parameters is observed; the UV/VIS data compares well with published reports [5]. With the exception of the data at $\mathrm{pH} 2.5$, midpoint temperature determined from return loss data is generally lower than that determined from UV/VIS absorbance; the average $T_{m}$ decrease is approximately $3{ }^{\circ} \mathrm{C}$. The enthalpy determined from return loss is also generally lower than that determined from UV/VIS absorbance. However, both sets of thermodynamic parameters yield good values of $\Delta \mathrm{C}_{\mathrm{p}}: 0.79 \mathrm{kcal} \mathrm{mol}^{-1} \mathrm{~K}^{-1}$ for the UV/VIS data and $1.44 \mathrm{kcal} \mathrm{mol}^{-1} \mathrm{~K}^{-1}$ for the return loss data. The published value of $\Delta \mathrm{C}_{\mathrm{p}}$ (assumed temperature-invariant) is $1.15 \mathrm{kcal} \mathrm{mol}^{-1} \mathrm{~K}^{-1}[5]$.

\section{CONCLUSIONS}

A coaxial-driven slot antenna has been demonstrated as a viable method for ultra-sensitive detection of changes in protein conformation. This system was used to assay the equilibrium thermal unfolding/refolding of RNase $A$. Return loss spectra were fitted to a series of Lorentzian peaks. The positions of selected Lorentzian peaks exhibited sigmoidal behavior with temperature. Thermodynamic parameters (midpoint temperature and van't Hoff enthalpy) obtained from return loss data are similar to those from UV/VIS absorbance.

Protein conformational change could be detected using the slot antenna at concentrations as low as $19 \mathrm{pM}$. This is nearly 6 orders of magnitude lower than the lowest concentration observable using UV/VIS absorbance (approximately $8.8 \mu \mathrm{M}$ ). Of the common techniques used for protein conformational studies, only fluorescence spectroscopy can be used at concentrations this low. Fluorescence spectroscopy requires either the presence of, or labeling of the protein with, suitable fluorescent agents. An advantage of our technique is that labeling is not required

TABLE I

VARIATION OF MIDPOINT TEMPERATURE AND ENTHALPY WITH PH

\begin{tabular}{|c|c|c|c|c|}
\hline \multirow{2}{*}{ pH } & \multicolumn{2}{|c|}{ UV/VIS } & \multicolumn{2}{c|}{ Return loss } \\
& $T_{m}\left({ }^{\circ} \mathbf{C}\right)$ & $\Delta \mathbf{H}_{m}(\mathbf{k c a l} / \mathbf{m o l})$ & $T_{m}\left({ }^{\circ} \mathbf{C}\right)$ & $\Delta \mathbf{H}_{m}(\mathbf{k c a l} / \mathbf{m o l})$ \\
\hline 2.5 & $39.21 \pm 0.30$ & $56.06 \pm 2.18$ & $42.12 \pm 1.54$ & $22.56 \pm 7.23$ \\
\hline 3.0 & $46.77 \pm 0.45$ & $53.20 \pm 0.61$ & $46.34 \pm 1.00$ & $63.02 \pm 4.15$ \\
\hline 3.5 & $54.01 \pm 0.64$ & $69.79 \pm 7.28$ & $53.13 \pm 2.69$ & $53.40 \pm 21.16$ \\
\hline 4.0 & $62.01 \pm 2.85$ & $70.73 \pm 18.24$ & $56.00 \pm 1.28$ & $64.94 \pm 11.49$ \\
\hline 4.5 & $63.56 \pm 0.76$ & $68.48 \pm 4.76$ & $60.25 \pm 5.53$ & $60.62 \pm 23.78$ \\
\hline 5.0 & $63.00 \pm 0.77$ & $76.44 \pm 10.31$ & $57.29 \pm 1.29$ & $51.43 \pm 12.15$ \\
\hline
\end{tabular}


for detection. Purified protein can be studied at low concentrations without the presence of potentially perturbing fluorescent agents. Midpoint temperature and enthalpy determined from return loss data are generally lower, and higher in error, than thermodynamic parameters obtained from UV/VIS data. The error, particularly in the enthalpy, is probably a result of uncertainty in the fitting of Lorentzian peaks. The lower $T_{m}$ and $\Delta H_{m}$ resulting from return loss data are noisy may be due to the differing nature of the phenomena being studied. UV/VIS absorbance at $288 \mathrm{~nm}$ assays changes in the absorbance of tyrosine residues, which are largely buried in the protein interior. Return loss measurements monitor changes in the dispersion of bound water, located on the protein surface. It is possible that the waters on the protein surface respond to protein unfolding at a lower temperature than do the hydrophobic molecules of the core. Specifically, the entropy of the hydrophilic surface residues may increase long before the unfolding of the core, resulting in a lower observed $\mathrm{T}_{\mathrm{m}}$ from dielectric measurements. The lower values of $\Delta \mathrm{H}_{m}$ may be explained if these changes in the entropy of surface residues extend over a wider temperature range than does unfolding of the hydrophobic core.

Future experiments include further testing of the system including additional biochemical experiments (such as chemical denaturation, enzyme kinetics and ligand binding) and also refinement and improvement of the antenna system.

\section{ACKNOWLEDGEMENTS}

Thanks to Alan Bettermann, Luke Palmer, Dr. John Peck and Pearl Wichaidit for help in antenna fabrication and to Dr. Charles Paulson for helpful suggestions. K.M.T. was supported by an NIH Molecular Biophysics Training Grant. This work is also supported by the Army Research Office and the Office of Naval Research.

\section{REFERENCES}

[1] R. Pethig, "Protein-water interactions determined by dielectric methods," Annu Rev Phys Chem, vol. 43, pp. 177-205, 1992.

[2] T. A. Klink, K. J. Woycechowsky, K. M. Taylor, and R. T. Raines, "Contribution of disulfide bonds to the conformational stability and catalytic activity of ribonuclease A," Eur J Biochem, vol. 267 , pp. 566-572, 2000.

[3] O. H. Lowry, N. J. Rosebrough, A. L. Farr, and R. J. Randall, "Protein measurement with the Folin phenol reagent," J. Biol. Chem., vol. 193, pp. 265-275, 1951.

[4] D. M. John and K. M. Weeks, "van't Hoff enthalpies without baselines," Protein Sci, vol. 9, pp. 1416-1419, 2000.

[5] C. N. Pace, G. R. Grimsley, S. T. Thomas, and G. I. Makhatadze, "Heat capacity change for ribonuclease A folding," Protein Sci., vol. 8, pp. 1500-1504, 1999. 